\title{
Human leukocyte antigen-DR/DQ eplet mismatch analysis for primary alloimmune risk stratification of non-sensitized kidney transplant recipients
}

\author{
Hyeyoung Lee ${ }^{1}$, Ji Won Min², Hyunhye Kang ${ }^{3}$, Chul Woo Yang ${ }^{4}$, Byung Ha Chung ${ }^{4}$, Eun-Jee $\mathrm{Oh}^{3}$

\begin{abstract}
${ }^{1}$ Department of Laboratory Medicine, International St. Mary's Hospital, Catholic Kwandong University College of Medicine, Incheon, Korea
${ }^{2}$ Division of Nephrology, Department of Internal Medicine, Bucheon St. Mary's Hospital, College of Medicine, The Catholic University of Korea, Bucheon, Korea ${ }^{3}$ Department of Laboratory Medicine, Seoul St. Mary's Hospital, College of Medicine, The Catholic University of Korea, Seoul, Korea

${ }^{4}$ Division of Nephrology, Department of Internal Medicine, Seoul St. Mary's Hospital, College of Medicine, The Catholic University of Korea, Seoul, Korea
\end{abstract}

Background: Human leukocyte antigen (HLA) analysis has been suggested as a predictive tool for alloimmune risk stratification in kidney transplantation (KT). We investigated whether the whole or single molecule HLA class II eplet mismatch was related to the de-novo donor-specific-antibody (dnDSA) development or antibody-mediated rejection (AMR).

Methods: Total 300 kidney transplants performed at Seoul St. Mary's Hospital between 2016 December and 2020 January without preformed DSA have been analyzed. HLA class II eplet mismatches were determined by high resolution HLA DRB1/DQB1 typing results and HLA Matchmaker program.

Results: During the 22.6 months (median) follow-up period, 24 recipients developed HLA class II dnDSA and 20 patients diagnosed as AMR. Using receiver operating characteristic analysis, we identified eplet mismatch thresholds associated with dnDSA development and stratified recipients into low-, intermediate-, and high-risk categories. In whole eplet mismatch analysis, risk categories were significantly correlated with dnDSA development (log-rank P-value $<0.001)$. High-risk group $(n=124)$ with HLADR 0-45 and HLA-DQ 8-31 significantly increased risk of dnDSA development compared to intermediate risk group with HLA-DR $\geq 14$ and HLA-DQ $\leq 7$, or HLA-DR 0-13 and HLA-DQ $7(n=86)(P=0.046)$. HLA class II eplet mismatches with HLA-DR 0-45 and HLA $\mathrm{DQ}>8$ predicted dnDSA development with $75 \%$ sensitivity and $63.4 \%$ specificity. Risk categories by single molecular eplet mismatch also had significant correlation with dnDSA development (log-rank P-value $=0.001$ ) with significant difference between low (HLA-DR $\leq 7$ and HLA-DQ $\leq 4)(n=78)$ and intermediate (HLA-DR $\geq 8$ and HLA-DQ $\leq 6$ or HLA-DR 0-7 and HLA-DQ 5-6) (n=95) group $(P=0.026)$. High-risk group (HLA-DR $0-20$ and HLA DQ 7-17) predicted dnDSA development with $75 \%$ sensitivity and $60.5 \%$ specificity. Both eplet mismatch risk categories were significantly associated with AMR, but no differences were found among risk groups.

Conclusions: HLA class II eplet mismatches showed the possibility of being used as a clinically relevant parameter in alloimmune risk assessments of $\mathrm{KT}$.

Corresponding author: Eun-Jee Oh

E-mail: ejoh@catholic.ac.kr

(C) The Korean Society for Transplantation

This is an Open Access article distributed under the terms of the Creative Commons Attribution Non-Commercial License (http://creativecommons.org/licenses/by-nc/4.0/) which permits unrestricted non-commercial use, distribution, and reproduction in any medium, provided the original work is properly cited. 